This item was submitted to Loughborough's Research Repository by the author.

Items in Figshare are protected by copyright, with all rights reserved, unless otherwise indicated.

\title{
Semi-infinite arrays of isotropic point scatterers. A unified approach
}

PLEASE CITE THE PUBLISHED VERSION

PUBLISHER

(C) Society for Industrial and Applied Mathematics

VERSION

VoR (Version of Record)

LICENCE

CC BY-NC-ND 4.0

REPOSITORY RECORD

Linton, C.M., and P.A. Martin. 2019. "Semi-infinite Arrays of Isotropic Point Scatterers. A Unified Approach". figshare. https://hdl.handle.net/2134/4267. 
This item was submitted to Loughborough's Institutional Repository (https://dspace.lboro.ac.uk/) by the author and is made available under the following Creative Commons Licence conditions.

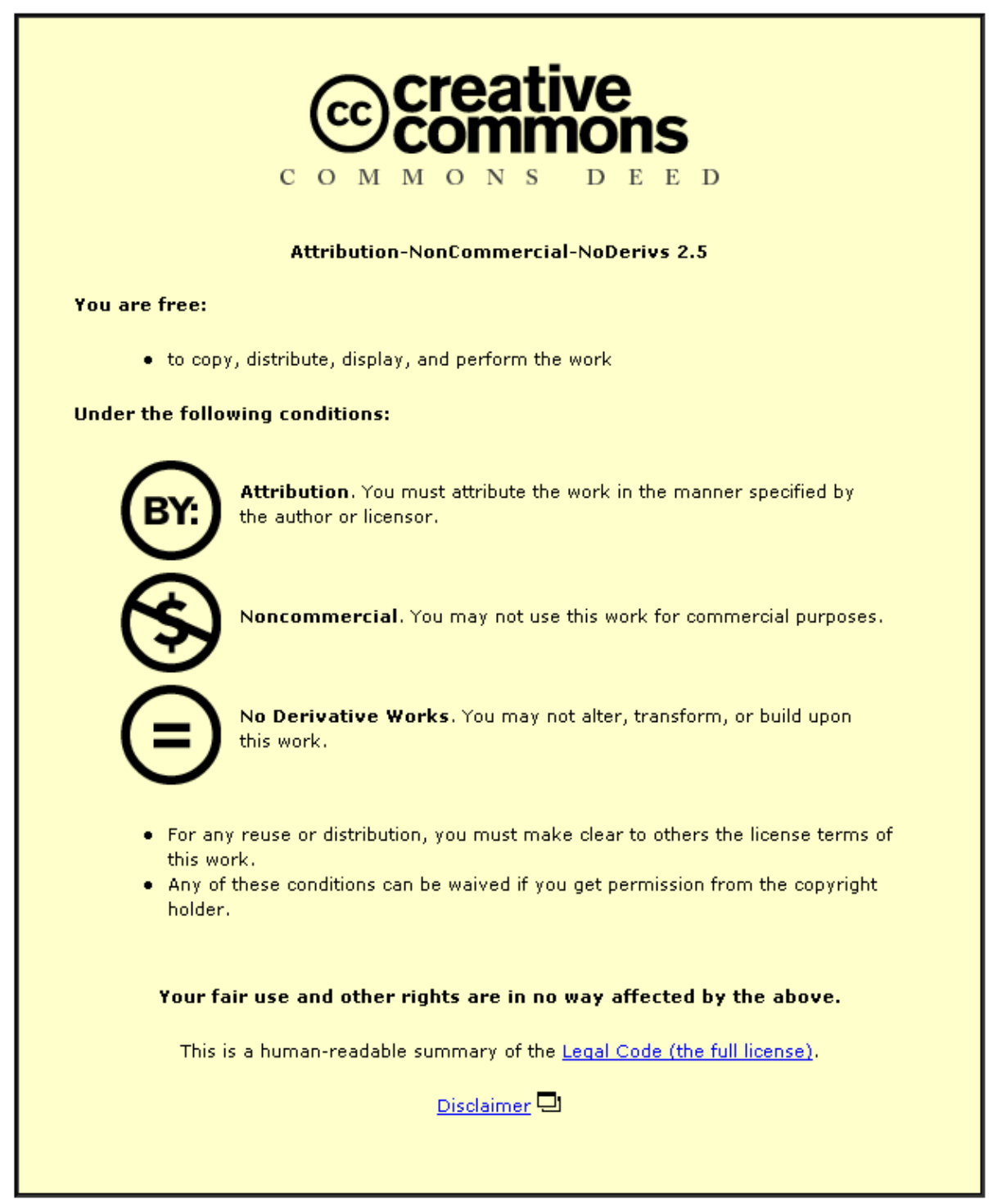

For the full text of this licence, please go to: http://creativecommons.org/licenses/by-nc-nd/2.5/ 


\title{
SEMI-INFINITE ARRAYS OF ISOTROPIC POINT SCATTERERS. A UNIFIED APPROACH*
}

\author{
C. M. LINTON ${ }^{\dagger}$ AND P. A. MARTIN ${ }^{\ddagger}$
}

\begin{abstract}
We solve the two-dimensional problem of acoustic scattering by a semi-infinite periodic array of identical isotropic point scatterers, i.e., objects whose size is negligible compared to the incident wavelength and which are assumed to scatter incident waves uniformly in all directions. This model is appropriate for scatterers on which Dirichlet boundary conditions are applied in the limit as the ratio of wavelength to body size tends to infinity. The problem is also relevant to the scattering of an $E$-polarized electromagnetic wave by an array of highly conducting wires. The actual geometry of each scatterer is characterized by a single parameter in the equations, related to the single-body scattering problem and determined from a harmonic boundary-value problem. Using a mixture of analytical and numerical techniques, we confirm that a number of phenomena reported for specific geometries are in fact present in the general case (such as the presence of shadow boundaries in the far field and the vanishing of the circular wave scattered by the end of the array in certain specific directions). We show that the semi-infinite array problem is equivalent to that of inverting an infinite Toeplitz matrix, which in turn can be formulated as a discrete Wiener-Hopf problem. Numerical results are presented which compare the amplitude of the wave diffracted by the end of the array for scatterers having different shapes.
\end{abstract}

Key words. scattering, semi-infinite array, Foldy's method, discrete Wiener-Hopf

AMS subject classifications. 74J20, 78A45

DOI. $10.1137 /$ S0036139903427891

1. Introduction. Many methods exist for studying wave interactions with finite arrays of scatterers. For some simple geometries, methods based on separation of variables can be used. For example, the scattering of a plane wave by an arbitrary finite array of circular cylinders can be reduced to the solution of a rapidly convergent infinite system of linear equations.

For more complicated geometries a different method is needed. One possibility is to express the solution to the multiple scattering problem in terms of the individual scattering characteristics of the elements that make up the array. This leads to the so-called $T$-matrix approach, which has been used extensively in acoustics and in other fields. Another technique is to formulate the problem as an integral equation by, for example, representing the solution as a distribution of dipoles over all the scatterers. This leads to an integral equation of the second kind for the unknown dipole strength. Discretization of the integral equations typically leads to large, full systems of algebraic equations.

As the size of an array increases, solutions to scattering problems rapidly become computationally expensive. In contrast, the case of an infinite periodic array excited by a plane wave is usually a much simpler proposition. This is because the periodicity allows us to formulate the problem on a single "cell" of the array, with periodic boundary conditions. In terms of integral equations this necessitates the use of a

${ }^{*}$ Received by the editors May 12, 2003; accepted for publication October 1, 2003; published electronically April 14, 2004.

http://www.siam.org/journals/siap/64-3/42789.html

${ }^{\dagger}$ Department of Mathematical Sciences, Loughborough University, Leicestershire LE11 3TU, UK (c.m.linton@lboro.ac.uk). The research of this author was supported in part by EPSRC Overseas Travel Grant GR/S31204/01.

${ }^{\ddagger}$ Department of Mathematical and Computer Sciences, Colorado School of Mines, Golden, CO 80401 (pamartin@mines.edu). 
more complicated Green's function, the efficient computation of which may be an issue; see [16].

Methods by which solutions to infinite array scattering problems can be applied to shed light on associated large finite array problems have been applied previously in the design of large phased array antennas, early examples using a Fourier windowing approach $[13,24]$, in which some fairly crude assumptions are made about the field near each scatterer. Recently, methods based on integral equations have been devised in which the basic idea is to formulate an integral equation for the difference between the infinite array and finite array solutions; see [26, 21], for example. For large finite one-dimensional arrays this leads to problems formulated on semi-infinite arrays. Associated with these so-called fringe integral equation methods is the analysis of Green's functions for semi-infinite arrays; see [5, 6, 17].

Scattering by a semi-infinite array is thus a problem of considerable interest from both a practical and a theoretical point of view, and very few results are available. Perhaps the only attempt at a general theory is that of Millar $[18,19]$ based on the analysis of a nonlinear integral equation. Some results have been derived previously for the case of small, widely spaced circular cylinders $[12,11]$ and for the strip grating at low frequencies $[22,23]$. In this paper we consider the general case of a semiinfinite array of identical scatterers which are each small with respect to the incident wavelength and under the assumption of Dirichlet boundary conditions on the scatterers. This problem was considered in [20], where a number of asymptotic results were derived. Our approach is based on Foldy's method [8], which since 1945 has found wide application in multiple scattering problems; for a recent application, see [2], for example. We show that the problems considered in [12, 11] and [22, 23] are special cases, and we construct a general system of equations in which the geometry of the scatterer is characterized by a single parameter. The system of equations can be inverted numerically or, since it is of Toeplitz type, it can be solved explicitly via the discrete Wiener-Hopf technique.

The semi-infinite array problem is closely related to the fully infinite array (i.e., diffraction grating) problem and, following a description of Foldy's method in section 2 , we next solve this for the same class of scatterers in section 3 . The semi-infinite grating problem is formulated in section 4 , including a detailed description of the form of the far field and of the behavior at resonance frequencies. Finally, in section 5 we show how the integral equation approach used in $[22,23]$ reduces to exactly the same equations as those found in section 4. Many of the technical details are relegated to the appendices.

2. Foldy's method. The classic work on acoustic scattering by semi-infinite gratings is that of Hills and Karp [12, 11], who consider small sound-soft circular cylinders. Their formulation is based on a technique due to Foldy [8]. Foldy considers isotropic point scatterers, meaning that "in the neighborhood of the $j$ th scatterer," the scattered field "will behave like" $A_{j} G\left(\mathbf{r}-\mathbf{r}_{j}\right)$, where the $j$ th scatterer is centered at $\mathbf{r}_{j}, A_{j}$ is an unknown amplitude, and $G$ is the free-space Green's function; in two dimensions $G(\mathbf{r})=H_{0}(k r)$, where $r=|\mathbf{r}|$ and $H_{0} \equiv H_{0}^{(1)}$ is a Hankel function. Foldy represents the total field as

$$
u(\mathbf{r})=u_{\mathrm{inc}}(\mathbf{r})+\sum_{j} A_{j} G\left(\mathbf{r}-\mathbf{r}_{j}\right),
$$


where the sum is over all the scatterers. The so-called external field is

$$
u_{n}(\mathbf{r}) \equiv u(\mathbf{r})-A_{n} G\left(\mathbf{r}-\mathbf{r}_{n}\right)=u_{\text {inc }}(\mathbf{r})+\sum_{\substack{j \\ j \neq n}} A_{j} G\left(\mathbf{r}-\mathbf{r}_{j}\right),
$$

which can be regarded as the "incident field" for the $n$th scatterer.

Now, characterize the scattering properties of the scatterers by

$$
A_{n}=f_{n} u_{n}\left(\mathbf{r}_{n}\right)
$$

where $f_{n}$ is "the scattering coefficient for the $n$th scatterer." Thus, the scattered field is determined by the value of the external field at the center of the scatterer, $\mathbf{r}_{n}$, together with the quantity $f_{n}$ (which we will come back to later). Then, (2.2) gives

$$
u_{n}(\mathbf{r})=u_{\mathrm{inc}}(\mathbf{r})+\sum_{\substack{j \\ j \neq n}} f_{j} u_{j}\left(\mathbf{r}_{j}\right) G\left(\mathbf{r}-\mathbf{r}_{j}\right) .
$$

Evaluating this equation at $\mathbf{r}_{n}$ gives, after using (2.3),

$$
f_{n}^{-1} A_{n}=u_{\text {inc }}\left(\mathbf{r}_{n}\right)+\sum_{\substack{j \\ j \neq n}} A_{j} G\left(\mathbf{r}_{n}-\mathbf{r}_{j}\right)
$$

which is a linear system of algebraic equations for the amplitudes $A_{j}$. The total field is then given by (2.1). When the scatterers are identical $f_{n}=f_{0}$, say. This quantity depends on the geometry of the scatterers and is discussed next.

The parameter $f_{0}$. The problem of scattering by a small sound-soft cylinder is a problem of low-frequency asymptotics. Using the general theory of Kleinman and Vainberg [14], we find that

$$
u_{\mathrm{sc}}(\mathbf{r}) \approx f_{0} u_{\mathrm{inc}}(\mathbf{0}) H_{0}(k r),
$$

where the origin is inside the cylinder's cross section $S$ and

$$
-\frac{1}{f_{0}}=\frac{2 \mathrm{i}}{\pi}(\ln k \ell-\delta)
$$

The complex constant $\delta$ occurs in the asymptotic approximation

$$
H_{0}(w)=\frac{2 \mathrm{i}}{\pi}(\ln w-\delta)+O\left(w^{2} \log w\right) \quad \text { as } w \rightarrow 0 ;
$$

thus, $\delta=\ln 2-C+\mathrm{i} \pi / 2$, where $C \approx 0.5772$ is Euler's constant. The length $\ell$ in (2.6) depends on the geometry of $S$. It is determined by solving the following twodimensional exterior Dirichlet problem for Laplace's equation: $\nabla^{2} v=0$ outside $S$, $v=0$ on $S$, and

$$
v=\ln (r / \ell)+o(1) \quad \text { as } r \rightarrow \infty .
$$

Thus, for an ellipse with semimajor axis $a$ and semiminor axis $b$, we obtain

$$
\ell=\frac{1}{2}(a+b)
$$


In particular, for circles of radius $a$, we have $\ell=a$, and then (2.6) is consistent with $-f_{0}^{-1}=H_{0}(k a)$, which is the approximation used by Hills and Karp [12]. Note that our formula for $f_{0},(2.6)$, does not depend on the orientation of $S$.

Let us make a few remarks. First, the approximation (2.5) is a rigorous, asymptotic approximation, valid for small sound-soft cylinders of any cross section. It is not merely a far-field result, but is valid in the near field too. It states that soft cylinders scatter isotropically - the scattered field does not depend on the direction of observation. None of these results is true for sound-hard cylinders (Neumann problem) or for penetrable cylinders (transmission problem), and thus Foldy's original method should be modified for nonsoft cylinders.

3. Infinite grating. We begin with the grating problem and consider the scattering of a plane wave

$$
u_{\text {inc }}=\mathrm{e}^{\mathrm{i}(\beta x+\alpha y)},
$$

where $\alpha=k \sin \psi$ and $\beta=k \cos \psi$, by an infinite row of identical scatterers, located at $(x, y)=(m s, 0), m=0, \pm 1, \pm 2, \ldots$, where $s$ is the spacing. We will use polar coordinates $\left(r_{m}, \theta_{m}\right)$ centered on the $m$ th scatterer and defined by

$$
x-m s=r_{m} \cos \theta_{m}, \quad y=r_{m} \sin \theta_{m},
$$

and we will write $(r, \theta)$ for $\left(r_{0}, \theta_{0}\right)$. In terms of $\left(r_{m}, \theta_{m}\right)$ the incident wave is

$$
u_{\text {inc }}=I_{m} \mathrm{e}^{\mathrm{i} k r_{m} \cos \left(\theta_{m}-\psi\right)}
$$

where

$$
I_{m}=\mathrm{e}^{\mathrm{i} \beta m s} .
$$

For future convenience we define the scattering angles $\psi_{m}, m=0, \pm 1, \pm 2, \ldots$, by

$$
\psi_{m}=\arccos \left(\frac{\beta_{m}}{k}\right), \quad \beta_{m}=\beta+\frac{2 m \pi}{s} .
$$

If $\left|\beta_{m}\right|<k$, i.e., if

$$
-1<\cos \psi+\frac{2 m \pi}{k s}<1
$$

we say that $m \in \mathcal{M}$ and then $0<\psi_{m}<\pi$. These correspond to the angles at which plane waves are scattered from an infinite grating; see (3.9) below. If $\left|\beta_{m}\right|>k$, then $\psi_{m}$ is no longer real and the appropriate branch of the arccos function is given by

$$
\arccos t= \begin{cases}\mathrm{i} \operatorname{arccosh} t, & t>1, \\ \pi-\mathrm{i} \operatorname{arccosh}(-t), & t<-1,\end{cases}
$$

with $\operatorname{arccosh} t=\ln \left(t+\sqrt{t^{2}-1}\right)$ for $t>1$.

Let us apply Foldy's method to the problem of the scattering of a plane wave by an infinite row of identical (small) sound-soft scatterers. The system (2.4) becomes (with $B_{n}$ as the unknowns)

$$
f_{0}^{-1} B_{n}=u_{\text {inc }}(n s, 0)+\sum_{\substack{m=-\infty \\ m \neq n}}^{\infty} B_{m} H_{0}(k s|m-n|), \quad n=0, \pm 1, \pm 2, \ldots
$$


We have $u_{\text {inc }}(m s, 0)=\mathrm{e}^{\mathrm{i} \beta s m}=I_{m}$, and quasi-periodicity (see (3.2)) gives $B_{m}=I_{m} B_{0}$, and then (3.4) gives

$$
-f_{0}^{-1} B_{0}+B_{0} \sum_{\substack{m=-\infty \\ m \neq n}}^{\infty} H_{0}(k s|m-n|) I_{m-n}=-1, \quad n=0, \pm 1, \pm 2, \ldots
$$

Hence, $B_{0}=\left(f_{0}^{-1}-\sigma(\psi)\right)^{-1}$, where

$$
\sigma(\psi)=\sum_{\substack{m=-\infty \\ m \neq n}}^{\infty} H_{0}(k s|m-n|) I_{m-n}=\sum_{j=1}^{\infty}\left(I_{j}+I_{-j}\right) H_{0}(k j s) .
$$

It will be convenient to define a quantity $\mathcal{K}$ by

$$
\mathcal{K}=-1 / B_{0}=\sigma(\psi)-f_{0}^{-1},
$$

so that $B_{n}=-I_{n} / \mathcal{K}$.

The far field. From (2.1)

$$
u=u_{\text {inc }}-\frac{1}{\mathcal{K}} \sum_{m=-\infty}^{\infty} I_{m} H_{0}\left(k r_{m}\right) .
$$

If we insert the integral representation (A.1), we get

$$
u=u_{\mathrm{i} n c}-\frac{1}{\mathcal{K}} \sum_{m=-\infty}^{\infty} \mathrm{e}^{\mathrm{i} \beta m s} \int_{-\infty}^{\infty} \frac{\mathrm{e}^{-k \gamma(t)|y|}}{\gamma(t)} \mathrm{e}^{\mathrm{i} k(x-m s) t} \mathrm{~d} t .
$$

Now use the Poisson summation formula (B.1) to get

$$
u=u_{\mathrm{inc}}-2 \sum_{m=-\infty}^{\infty} \frac{\mathrm{e}^{\mathrm{i} k r \cos \left(\theta-\operatorname{sgn}(y) \psi_{m}\right)}}{k s \mathcal{K} \sin \psi_{m}},
$$

where we have used $\gamma\left(\beta_{m} / k\right)=-\mathrm{i} \sin \psi_{m}$. Note that $-\mathrm{i} \psi_{m}$ is real and positive if $\left|\beta_{m} / k\right|>1$, and so the terms in the sum for these values of $m$ decay as $|y| \rightarrow \infty$. The far field involves only those $m$ for which $m \in \mathcal{M}$ and thus, as $y \rightarrow \pm \infty$,

$$
u \sim u_{\mathrm{i} n c}-2 \sum_{m \in \mathcal{M}} \frac{\mathrm{e}^{\mathrm{i} k r \cos \left(\theta \mp \psi_{m}\right)}}{k s \mathcal{K} \sin \psi_{m}} .
$$

The scattered field, which is symmetric about the $x$-axis, thus consists of a number of plane waves, that number increasing as $k s$ increases. In $y>0$, these waves make angles $\psi_{m}$ with the positive $x$-axis. For sufficiently small $k s$ there is just one plane wave corresponding to $m=0$.

Resonance. For large $j$ we have

$$
\left(I_{j}+I_{-j}\right) H_{0}(k j s) \sim\left(\mathrm{e}^{\mathrm{i} j \beta s}+\mathrm{e}^{-\mathrm{i} j \beta s}\right) \sqrt{\frac{2}{\pi j k s}} \mathrm{e}^{\mathrm{i}\left(k j s-\frac{1}{4} \pi\right)},
$$

and so the sum in (3.6) fails to converge if $(k \pm \beta) s=2 n \pi$ for some integer $n$. This condition corresponds to $\beta_{n}= \pm k$ for some integer $n$, which implies that $\psi_{n}=0$ or 
$\psi_{n}=\pi$. An alternative expression for $\sigma(\psi)$ is given in (C.1), which shows that as $\psi_{n}$ approaches 0 or $\pi$,

$$
\mathcal{K} \sim \sigma(\psi) \sim 2\left(k s \sin \psi_{n}\right)^{-1}
$$

Such a situation is termed resonance, and all the coefficients $B_{m}$ tend to zero in this limit. The field is not zero, though. Indeed, from (3.8) we have

$$
u=u_{\text {inc }}-\mathrm{e}^{\mathrm{i} k x \cos \psi_{n}}
$$

in this limit, so that the scattered field reduces to a wave propagating along the grating, either towards $x=\infty\left(\psi_{n}=0\right)$ or towards $x=-\infty\left(\psi_{n}=\pi\right)$. For simplicity, we will exclude the possibility that $k s$ is an integer multiple of $\pi$ so that we cannot satisfy $\cos \psi_{n}=-1$ and $\cos \psi_{m}=1$ simultaneously.

4. Semi-infinite grating. Suppose now that we have a semi-infinite grating of scatterers located along the positive $x$-axis at $(x, y)=(m s, 0), m=0,1,2, \ldots$. Again, as the scatterers are identical, we have $f_{m} \equiv f_{0}$. Then, the scattered field is given from (2.1) by

$$
u_{\mathrm{sc}}=\sum_{n=0}^{\infty} A_{n} H_{0}\left(k r_{n}\right),
$$

where the coefficients $A_{n}$ are found to satisfy

$$
A_{n}-f_{0} \sum_{\substack{m=0 \\ m \neq n}}^{\infty} A_{m} H_{0}(k s|m-n|)=f_{0} I_{n}, \quad n=0,1,2, \ldots,
$$

which is equivalent to [12, (3.1-1)] (apart from a missing $A_{n}$ ) and [20, (41)]. If we write $A_{n}=I_{n} B_{0}+C_{n}$, where $B_{0}=-\mathcal{K}^{-1}$ is the solution to the corresponding infinite grating problem, then we find that, for $n=0,1,2, \ldots$,

$$
C_{n}-f_{0} \sum_{\substack{m=0 \\ m \neq n}}^{\infty} C_{m} H_{0}(k s|m-n|)=\frac{f_{0}}{\mathcal{K}} \sum_{j=n+1}^{\infty} I_{n-j} H_{0}(k j s) .
$$

Both (4.2) and (4.3) can be written in terms of Toeplitz matrices, which can be inverted either directly using numerical truncation or via the discrete Wiener-Hopf technique as described in Appendix E. Note that in order to compute the slowly convergent sum on the right-hand side of (4.3) we use (C.2).

For large $n$, the sum over $j$ on the right-hand side of (4.3) satisfies

$$
\sum_{j=n+1}^{\infty} I_{n-j} H_{0}(k j s) \sim-\sqrt{\frac{2}{\pi k n s}} \frac{\mathrm{e}^{-\frac{1}{4} \mathrm{i} \pi} \mathrm{e}^{\mathrm{i} k s n}}{\left[1-(-1)^{q} \mathrm{e}^{-\mathrm{i}(k-\beta) s / 2}\right]},
$$

where we have used the asymptotic form for the Hankel function with large argument and (D.1), and $q$ is such that $2 q \pi<(k-\beta) s<2(q+1) \pi$ (i.e., $\beta_{q}<k<\beta_{q+1}$ ). One might expect, therefore, that $C_{n}=O\left(n^{-1 / 2}\right)$ as $n \rightarrow \infty$. In fact, calculations show that $C_{n}=O\left(n^{-3 / 2}\right)$ as $n \rightarrow \infty$. The same decay rate is observed in [22] and is consistent with behavior in the equivalent half-plane problem. The reason for this faster-than-expected decay is the presence of the phase exp(iksn) in the large $n$ behavior of the right-hand side; see (E.8), (E.10), and (E.12). 
The far field. The approximation $C_{m}=0$ leads to what is known as the Kirchhoff solution. In this case, (4.1) becomes, after the substitution of the integral representation for the Hankel function given by (A.1) and use of (B.2),

$$
u_{\mathrm{sc}}^{\mathrm{K}}=\frac{\mathrm{i}}{\pi \mathcal{K}} \int_{-\infty}^{\infty} \frac{\mathrm{e}^{-k \gamma(t)|y|}}{\gamma(t)} \frac{\mathrm{e}^{\mathrm{i} k x t}}{1-\mathrm{e}^{\mathrm{i} s(\beta-k t)}} \mathrm{d} t .
$$

The total field is given by

$$
u_{\mathrm{sc}}=u_{\mathrm{sc}}^{\mathrm{K}}-\frac{\mathrm{i}}{\pi} \int_{-\infty}^{\infty} \frac{\mathrm{e}^{-k \gamma(t)|y|}}{\gamma(t)} \mathrm{e}^{\mathrm{i} k x t} \sum_{n=0}^{\infty} C_{n} \mathrm{e}^{-\mathrm{i} k n s t} \mathrm{~d} t .
$$

From (F.1), for $-\pi<\theta<\pi$,

$$
u_{\mathrm{sc}}^{\mathrm{K}}(r, \theta) \sim \frac{\mathrm{e}^{\mathrm{i}\left(k r-\frac{1}{4} \pi\right)}}{\mathcal{K}\left(\mathrm{e}^{\mathrm{i} k s(\cos \psi-\cos \theta)}-1\right)} \sqrt{\frac{2}{\pi k r}}-\sum_{\substack{m \in \mathcal{M} \\ \psi_{m}>|\theta|}} \frac{2 \mathrm{e}^{\mathrm{i} k r \cos \left(|\theta|-\psi_{m}\right)}}{k s \mathcal{K} \sin \psi_{m}} \quad \text { as } k r \rightarrow \infty,
$$

with the addition of the correction term $\tilde{I}$ given by (F.2) when $|\theta|$ is close to $\psi_{p}$, and

$$
u_{\mathrm{sc}} \sim u_{\mathrm{sc}}^{\mathrm{K}}+\sqrt{\frac{2}{\pi k r}} \mathrm{e}^{\mathrm{i}\left(k r-\frac{1}{4} \pi\right)} \sum_{n=0}^{\infty} C_{n} \mathrm{e}^{-\mathrm{i} k n s \cos \theta} .
$$

Just as in the infinite grating problem, the scattered field is symmetric about the $x$-axis. To simplify the discussion of the far field we will assume that $y>0$ (i.e., $0<\theta<\pi)$. If we define

$$
\tilde{H}(k r)=\sqrt{\frac{2}{\pi k r}} \mathrm{e}^{\mathrm{i}\left(k r-\frac{1}{4} \pi\right)}
$$

and

$$
g(\theta, \psi)=\frac{1}{\mathcal{K}\left(\mathrm{e}^{\mathrm{i} k s(\cos \psi-\cos \theta)}-1\right)}+\sum_{n=0}^{\infty} C_{n} \mathrm{e}^{-\mathrm{i} k n s \cos \theta},
$$

then we have

$$
u_{\mathrm{sc}} \sim g(\theta, \psi) \tilde{H}(k r)-\sum_{\substack{m \in \mathcal{M} \\ \psi_{m}>\theta}} \frac{2 \mathrm{e}^{\mathrm{i} k r \cos \left(\theta-\psi_{m}\right)}}{k s \mathcal{K} \sin \psi_{m}} .
$$

The far field thus consists of a circular wave of "amplitude" $g(\theta, \psi)$ and a set of plane waves. These plane waves propagate in the same directions as for the infinite grating but do not exist everywhere. The plane wave making an angle $\psi_{m}$ with the positive $x$-axis is found only in the sector $\theta<\psi_{m}$. It is apparent that the coefficients $C_{n}$ affect only the circular wave, but that the plane wave field is determined solely by the Kirchhoff solution. In the numerical results presented below we will thus focus only on the circular wave.

The lines $\theta=\psi_{m}$ are known as shadow boundaries, and the circular wave becomes infinite as the shadow boundaries are approached. In fact near these lines we should add a term to $g(\theta, \psi)$ given from (F.2) by

$$
\tilde{g}(r, \theta, \psi)=\frac{\mathrm{i}\left(1+2 \mathrm{i} \zeta_{p} \mathrm{e}^{-\mathrm{i} \zeta_{p}^{2}} F\left(\zeta_{p}\right)\right)}{2 k s \mathcal{K} \sin \frac{1}{2}\left(\theta-\psi_{p}\right) \sin \psi_{p}},
$$




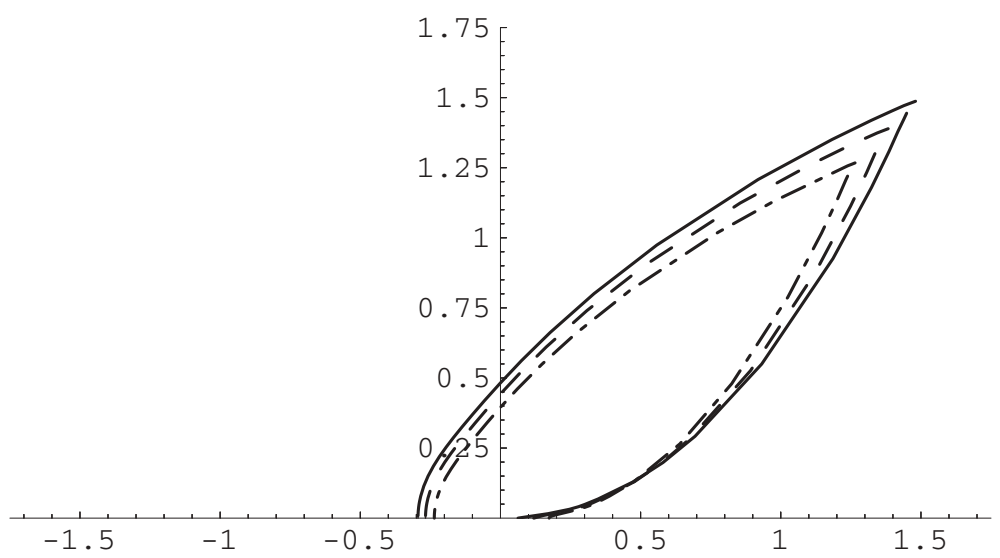

FIG. 4.1. The amplitude of the circular wave, $|g(\theta, \psi)+\tilde{g}(r, \theta, \psi)|$, for three different scatterers when $k a=0.05, k s=2, k r=20$, and $\psi=\pi / 4$. The solid line corresponds to an array of circles of radius $a$, the dashed line corresponds to ellipses with $a / b=2$, and the dash-dot line corresponds to plates of length $2 a$.

where $\zeta_{p}=\sqrt{2 k r} \sin \frac{1}{2}\left|\theta-\psi_{p}\right|$ and $F$ is the Fresnel integral defined in (F.3). The combination $g+\tilde{g}$ is bounded as $\theta \rightarrow \psi_{p}$ for any $r$, but the limit is different from each side. Since $F(0)=\frac{1}{2} \sqrt{\pi} \exp (\mathrm{i} \pi / 4)$, the discontinuity in $g+\tilde{g}$ as $\theta$ passes through $\psi_{p}$ exactly cancels the extra residue contribution that appears in the sum in (4.7) as the shadow boundary is crossed.

Hills and Karp [12] introduced the characteristic angles $\psi_{m}(0)$. These are the real scattering angles when the incident wave angle is zero, i.e.,

$$
\psi_{m}(0)=\arccos \left(1+\frac{2 m \pi}{k s}\right), \quad m=-[k s / \pi], \ldots,-1,0 .
$$

It follows from (4.6) and (E.13) that in the full solution the circular wave vanishes in these directions, i.e., $g\left(\psi_{m}(0), \psi\right)=0$. Hills and Karp state this result only for large $k s$, but it is true for any value of $k s$, provided that the Wiener-Hopf factorization described in Appendix E exists. Calculations in [22] suggest that this result is true for moderate values of $k s$ (about 3.5) but perhaps not for small $k s$ (about 0.7). Our calculations based on (4.3) indicate that $g(0, \psi)=0$ for all $k s$. (For $k s<\pi$ there is only one characteristic angle, namely, $\theta=0$.)

In Figures 4.1-4.3 we show as polar plots the amplitude of the circular wave, $|g(\theta, \psi)+\tilde{g}(r, \theta, \psi)|$, for three different scatterers when $k a=0.05, \psi=\pi / 4$, and $k r=20$. In Figure 4.1, $k s=2$ and the scatterers are fairly close together, whereas in Figure 4.3, $k s=10$ and the scatterers are well separated. Figure 4.2 represents an intermediate case with $k s=5$. In each case the three different (discontinuous) curves correspond to an array of circles of radius $a$ (solid lines), ellipses with semimajor axis $a$ and semiminor axis $\frac{1}{2} a$ (dashed lines), and plates of length $2 a$ (dash-dot lines). The quantity $f_{0}$ is calculated from (2.6) in each case with $\ell=a, \frac{3}{4} a$, and $\frac{1}{2} a$, respectively.

It can be seen that the general form of each of the curves is the same but that circles produce the diffracted wave with the largest amplitude, while the plates produce the smallest effect. The scales on the three figures are not the same, and it is clear that the amplitude of the diffracted wave generally diminishes as $k s$ increases (though not necessarily for a given observation angle). The numerical results were obtained by direct truncation of (4.3) and checked against (E.10). In order to represent the zeros 


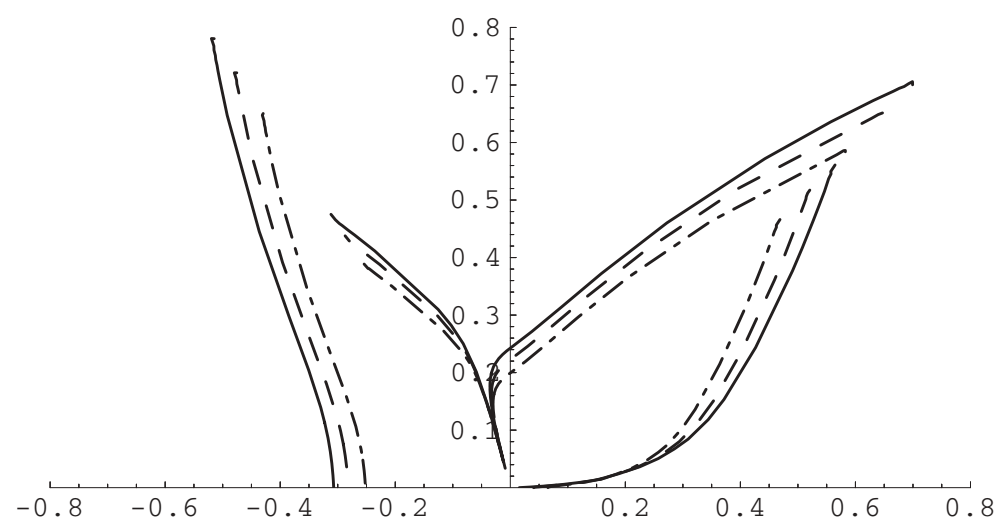

FIG. 4.2. The amplitude of the circular wave, $|g(\theta, \psi)+\tilde{g}(r, \theta, \psi)|$, for three different scatterers when $k a=0.05, k s=5, k r=20$, and $\psi=\pi / 4$. The solid line corresponds to an array of circles of radius $a$, the dashed line corresponds to ellipses with $a / b=2$, and the dash-dot line corresponds to plates of length $2 a$.

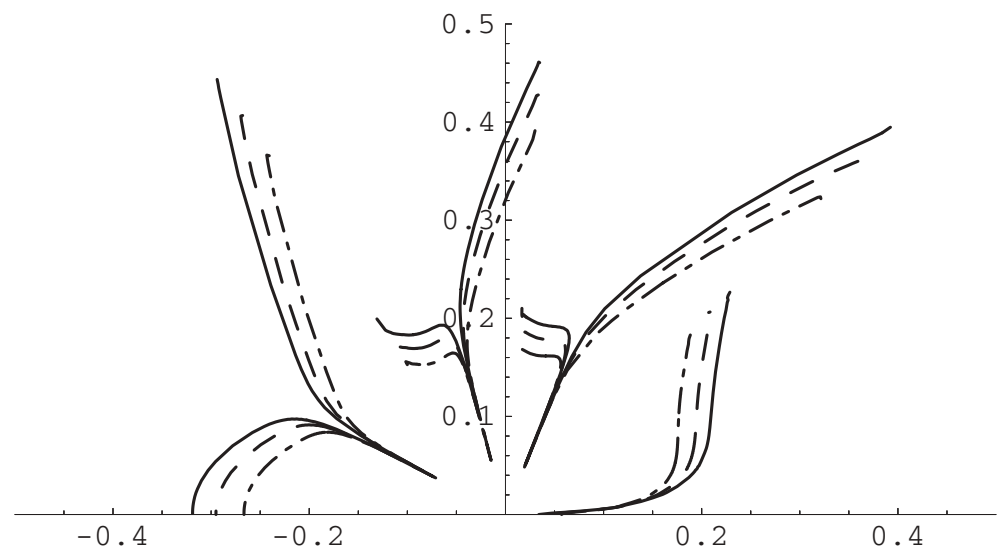

FIG. 4.3. The amplitude of the circular wave, $|g(\theta, \psi)+\tilde{g}(r, \theta, \psi)|$, for three different scatterers when $k a=0.05, k s=10, k r=20$, and $\psi=\pi / 4$. The solid line corresponds to an array of circles of radius $a$, the dashed line corresponds to ellipses with $a / b=2$, and the dash-dot line corresponds to plates of length $2 a$.

in the directions $\psi_{m}(0)$ with reasonable accuracy, a $200 \times 200$ system of equations was used, though the main features of the solution are accurately represented if a much smaller truncation is used.

In Figure 4.1 there is just one predominant scattering direction corresponding to the direction of the incident wave, and the amplitude of the wave is zero for $\theta=$ $\psi_{0}(0)=0$. In Figure 4.2 there are two predominant scattering directions corresponding to $\psi_{0}=\pi / 4$ and $\psi_{-1}=\arccos (1 / \sqrt{2}-2 \pi / 5) \approx 0.685 \pi$. The amplitude of the wave is zero in the directions $\psi_{0}(0)=0$ and $\psi_{-1}(0)=\arccos (1-2 \pi / 5) \approx 0.583 \pi$. In Figure 4.3 there are three predominant scattering directions corresponding to $\psi_{0}=\pi / 4$, $\psi_{-1}=\arccos (1 / \sqrt{2}-\pi / 5) \approx 0.475 \pi$, and $\psi_{-2}=\arccos (1 / \sqrt{2}-2 \pi / 5) \approx 0.685 \pi$. The amplitude of the wave is zero in the directions $\psi_{0}(0)=0, \psi_{-1}(0)=\arccos (1-\pi / 5) \approx$ $0.379 \pi, \psi_{-2}(0)=\arccos (1-2 \pi / 5) \approx 0.583 \pi$, and $\psi_{-3}(0)=\arccos (1-3 \pi / 5) \approx 0.846 \pi$. 
Inward and outward resonance. For fixed $n$ the sum on the right-hand side of (4.3) converges unless $(k-\beta) s=2 m \pi$ for some integer $m$ (in other words, unless $\psi_{m}=0$ for some integer $m$ ). Following Hills and Karp [12], we call this inward resonance. We also use the term outward resonance for the case when $\psi_{m}=\pi$ for some integer $m$. In either situation $1 / \mathcal{K}=0$. Provided we do not have inward resonance, $C_{n} \rightarrow 0$ as $n \rightarrow \infty$.

For outward resonance, we get simply $C_{n}=0$ and

$$
u_{\mathrm{sc}}=u_{\mathrm{sc}}^{\mathrm{K}}=-\frac{1}{\mathcal{K}} \sum_{m=0}^{\infty} I_{m} H_{0}\left(k r_{m}\right)=\frac{\mathrm{i}}{\pi \mathcal{K}} \int_{-\infty}^{\infty} \frac{\mathrm{e}^{-k \gamma(t)|y|}}{\gamma(t)} \frac{\mathrm{e}^{\mathrm{i} k x t}}{1-\mathrm{e}^{\mathrm{i} s(\beta-k t)}} \mathrm{d} t .
$$

As $\psi_{n} \rightarrow \pi$ we have $\mathcal{K} \sim 2 /\left(k s \sin \psi_{n}\right)$, and in order to find the singular behavior of the integral in this limit (which corresponds to one of the poles of the integrand coinciding with the branch point of $\gamma$ at $t=-1$; the branch cut extending to $-\mathrm{i} \infty$ ) we deform the contour so that it passes above the pole at $\beta_{n} / k$ and thus pick up a contribution $2 \pi \mathrm{ie}^{-\mathrm{i} k x} /\left(k s \sin \psi_{n}\right)$, the remaining integral being finite. Thus, for outward resonance, we obtain

$$
u_{\mathrm{sc}}=-\mathrm{e}^{-\mathrm{i} k x}
$$

in agreement with $[12,(3.6-1)]$.

In the inward resonance case $\psi_{n} \rightarrow 0$, the same arguments show that the Kirchhoff solution $u_{\mathrm{sc}}^{\mathrm{K}}$ approaches $-\mathrm{e}^{\mathrm{i} k x}$, but the region of existence shrinks to the line $\theta=0$, and this no longer represents the total scattered field; see [11]. We have not considered the case where inward and outward resonance occur together (which can only happen if $k s$ is an integer multiple of $\pi$ ). Some results for this case can be found in [20].

5. Semi-infinite strip grating. Here we will demonstrate that the analysis given in $[22,23]$ for a semi-infinite strip grating can be reduced to the general form given in section 4. Consider first an infinite set of strips $S_{n}=(n s-a, n s+a)$, $n=0, \pm 1, \pm 2, \ldots(s>2 a)$, on which we have $u=0$ and write $S=\bigcup_{n=-\infty}^{\infty} S_{n}$. We wish to solve the Helmholtz equation in $y>0$ with

$$
\begin{aligned}
\frac{\partial u_{\mathrm{sc}}}{\partial y} & =0 & & \text { on } y=0, x \notin S, \\
u_{\mathrm{sc}} & =-\mathrm{e}^{\mathrm{i} \beta x} & & \text { on } y=0, x \in S .
\end{aligned}
$$

Define $v_{\mathrm{sc}}(x) \equiv \partial u_{\mathrm{sc}} /\left.\partial y\right|_{y=0}$. Then we have the integral equation

$$
\int_{S} v_{\text {sc }}(\xi) G(x-\xi, 0) \mathrm{d} \xi=-\mathrm{e}^{\mathrm{i} \beta x}, \quad x \in S,
$$

where $G(X, Y)=-\frac{1}{2} \mathrm{i} H_{0}\left(k \sqrt{X^{2}+Y^{2}}\right)$ is the free-space Green's function. Equivalently, since $v_{\mathrm{sc}}(\xi+m s)=I_{m} v_{\mathrm{sc}}(\xi)$,

$$
\sum_{m=-\infty}^{\infty} I_{m} \int_{-a}^{a} v_{\mathrm{sc}}(\xi) G(x-\xi-m s, 0) \mathrm{d} \xi=-\mathrm{e}^{\mathrm{i} \beta x}, \quad|x|<a,
$$

which can be written

$$
\int_{-a}^{a} v_{\mathrm{sc}}(\xi) G_{\beta}(x-\xi, 0) \mathrm{d} \xi=-\mathrm{e}^{\mathrm{i} \beta x}, \quad|x|<a,
$$


where we have defined

$$
G_{\beta}(X, Y)=\sum_{m=-\infty}^{\infty} I_{m} G(X-m s, Y)=-\frac{\mathrm{i}}{k s} \sum_{m=-\infty}^{\infty} \frac{\mathrm{e}^{\mathrm{i} k|Y| \sin \psi_{m}} \mathrm{e}^{\mathrm{i} \beta_{m} X}}{\sin \psi_{m}}
$$

using (B.1). The scattered field is then represented by

$$
u_{\mathrm{sc}}(x, y)=\int_{S} v_{\mathrm{sc}}(\xi) G(x-\xi, y) \mathrm{d} \xi=\int_{-a}^{a} v_{\mathrm{sc}}(\xi) G_{\beta}(x-\xi, y) \mathrm{d} \xi .
$$

Now consider a semi-infinite set of strips $S_{n}=(n s-a, n s+a), n=0,1,2, \ldots$ $(s>2 a)$, and write $S^{+}=\bigcup_{n=-\infty}^{\infty} S_{n}$. We have

$$
\int_{S^{+}} v_{\mathrm{sc}}^{+}(\xi) G(x-\xi, 0) \mathrm{d} \xi=-\mathrm{e}^{\mathrm{i} \beta x}, \quad x \in S^{+},
$$

and

$$
u_{\mathrm{sc}}^{+}(x, y)=\int_{S^{+}} v_{\mathrm{sc}}^{+}(\xi) G(x-\xi, y) \mathrm{d} \xi .
$$

Write $u_{\mathrm{sc}}^{+}=\phi+u_{\mathrm{sc}}$ and $\nu(x) \equiv \partial \phi /\left.\partial y\right|_{y=0}$. Then the integral equation becomes

$\sum_{m=0}^{\infty} \int_{-a}^{a} \nu(\xi+m s) G(x-\xi-m s, 0) \mathrm{d} \xi+\int_{-a}^{a} v_{\mathrm{sc}}(\xi) G_{\beta}^{+}(x-\xi, 0) \mathrm{d} \xi=-\mathrm{e}^{\mathrm{i} \beta x}, \quad x \in S^{+}$,

where we have defined

$$
G_{\beta}^{+}(X, Y)=\sum_{m=0}^{\infty} I_{m} G(X-m s, Y)=-\frac{1}{2 \pi} \int_{-\infty}^{\infty} \frac{\mathrm{e}^{-k \gamma(t)|Y|}}{\gamma(t)} \frac{\mathrm{e}^{\mathrm{i} k X t}}{1-\mathrm{e}^{\mathrm{i} s(\beta-k t)}} \mathrm{d} t,
$$

using (B.2). Equation (5.3) is the starting point for the numerical calculations given in $[22,23]$. Alternatively, using (5.1),

$$
\sum_{m=0}^{\infty} \int_{-a}^{a} \nu(\xi+m s) G(x-\xi-m s, 0) \mathrm{d} \xi=\int_{-a}^{a} v_{\mathrm{sc}}(\xi) G_{\beta}^{-}(x-\xi, 0) \mathrm{d} \xi, \quad x \in S^{+},
$$

where $G_{\beta}^{-}=G_{\beta}-G_{\beta}^{+}$.

Under the assumption that $k a \ll 1$, we make the approximations (as in [22, 23])

$$
v_{\mathrm{sc}}(x)=\frac{2 \mathrm{i} B}{\pi \sqrt{a^{2}-x^{2}}}, \quad \nu(x+m s)=\frac{2 \mathrm{i} C_{m}}{\pi \sqrt{a^{2}-x^{2}}}, \quad m=0,1,2, \ldots,
$$

with $|x|<a$ in all cases. To determine $B$ we average (5.1) so that

$$
\frac{2 \mathrm{i} B}{\pi} \int_{-a}^{a} \int_{-a}^{a} \frac{G_{\beta}(x-\xi, 0)}{\sqrt{a^{2}-\xi^{2}}} \mathrm{~d} \xi \mathrm{d} x=-\int_{-a}^{a} \mathrm{e}^{\mathrm{i} \beta x} \mathrm{~d} x=-\frac{2}{\beta} \sin \beta a \approx-2 a
$$

or

$$
B=-\left(\sum_{m=-\infty}^{\infty} I_{m} \mathcal{G}_{m}\right)^{-1}
$$


where we have defined

$$
\mathcal{G}_{n}=\frac{\mathrm{i}}{\pi a} \int_{-a}^{a} \int_{-a}^{a} \frac{G(x-\xi-n s, 0)}{\sqrt{a^{2}-\xi^{2}}} \mathrm{~d} \xi \mathrm{d} x
$$

for which approximate values, valid for $k a \ll 1$, can be derived; see (G.1) and (G.2).

To determine $C_{m}$ we average (5.5) so that for each $n=0,1,2, \ldots$

$$
\sum_{m=0}^{\infty} C_{m} \int_{n s-a}^{n s+a} \int_{-a}^{a} \frac{G(x-\xi-m s, 0)}{\sqrt{a^{2}-\xi^{2}}} \mathrm{~d} \xi \mathrm{d} x=B \int_{n s-a}^{n s+a} \int_{-a}^{a} \frac{G_{\beta}^{-}(x-\xi, 0)}{\sqrt{a^{2}-\xi^{2}}} \mathrm{~d} \xi \mathrm{d} x
$$

or

$$
\sum_{m=0}^{\infty} C_{m} \mathcal{G}_{m-n}=B \sum_{m=-\infty}^{-1} I_{m} \mathcal{G}_{m-n}
$$

which, once $B$ is determined from (5.6), is an infinite system of algebraic equations for the unknowns.

If we substitute the approximate values for $\mathcal{G}_{n}(n>0)$ from (G.2), we get $B=$ $-1 /\left(\mathcal{G}_{0}+\sigma(\psi)\right)$, and then

$$
C_{n}+\frac{1}{\mathcal{G}_{0}} \sum_{\substack{m=0 \\ \neq n}}^{\infty} C_{m} H_{0}(k|n-m| s)=-\frac{1 / \mathcal{G}_{0}}{\mathcal{G}_{0}+\sigma(\psi)} \sum_{j=n+1}^{\infty} I_{n-j} H_{0}(k j s),
$$

which is of exactly the same form as (4.3), since $\mathcal{G}_{0}=-1 / f_{0}$ (compare (G.1) and (2.6) with $\ell=a / 2$ ).

The field is then given, from (5.2), by

$$
\begin{aligned}
u_{\mathrm{sc}}^{+}(x, y) & =\int_{S^{+}}\left(\nu(\xi)+v_{\mathrm{sc}}(\xi)\right) G(x-\xi, y) \mathrm{d} \xi \\
& =\frac{2 \mathrm{i}}{\pi} \sum_{m=0}^{\infty} C_{m} \int_{-a}^{a} \frac{G(x-\xi-m s, y)}{\sqrt{a^{2}-\xi^{2}}} \mathrm{~d} \xi+\frac{2 \mathrm{i} B}{\pi} \int_{-a}^{a} \frac{G_{\beta}^{+}(x-\xi, y)}{\sqrt{a^{2}-\xi^{2}}} \mathrm{~d} \xi .
\end{aligned}
$$

The last integral is, using (5.4),

$$
-\frac{1}{2 \pi} \int_{-\infty}^{\infty} \int_{-a}^{a} \frac{\mathrm{e}^{-\mathrm{i} k \xi t} \mathrm{~d} \xi}{\sqrt{a^{2}-\xi^{2}}} \frac{\mathrm{e}^{\mathrm{i} k x t-k \gamma(t)|y|}}{\gamma(t)\left(1-\mathrm{e}^{\mathrm{i} s(\beta-k t)}\right)} \mathrm{d} t=-\frac{1}{2} \mathcal{f}_{-\infty}^{\infty} \frac{J_{0}(k a t) \mathrm{e}^{\mathrm{i} k x t-k \gamma(t)|y|}}{\gamma(t)\left(1-\mathrm{e}^{\mathrm{i} s(\beta-k t)}\right)} \mathrm{d} t
$$

and similarly for the first integral so that

$$
\begin{aligned}
u_{\mathrm{sc}}^{+}(x, y)= & -\frac{\mathrm{i}}{\pi} \sum_{m=0}^{\infty} C_{m} \int_{-\infty}^{\infty} \frac{\mathrm{e}^{-k \gamma(t)|y|}}{\gamma(t)} J_{0}(k a t) \mathrm{e}^{\mathrm{i} k(x-m s) t} \mathrm{~d} t \\
& -\frac{\mathrm{i} B}{\pi} \oint_{-\infty}^{\infty} \frac{\mathrm{e}^{-k \gamma(t)|y|}}{\gamma(t)} \frac{J_{0}(k a t) \mathrm{e}^{\mathrm{i} k x t}}{1-\mathrm{e}^{\mathrm{i} s(\beta-k t)}} \mathrm{d} t,
\end{aligned}
$$

which, via the results in Appendix $\mathrm{F}$ and utilizing the fact that $k a \ll 1$, leads to precisely the same far-field asymptotics as that given in (4.5).

Note that exactly the same far-field is generated for a semi-infinite array of angled plates, since $f_{0}$ is independent of the plate orientation. Indeed, the plates in the array may all be oriented in different directions. 
6. Conclusion. Using a mixture of analysis (discrete Wiener-Hopf) and computation, we have studied the problem of acoustic scattering by a semi-infinite periodic array of identical isotropic point scatterers, i.e., scatterers which are small compared to the incident wavelength and on which Dirichlet boundary conditions are applied. The actual geometry is characterized by a single parameter in the equations, related to the single-body scattering problem. Numerical results have been presented which show the effect of the shape of the scatterer on the form of the circular wave diffracted by the end of the array.

Computations for semi-infinite arrays under less restrictive assumptions than that of isotropic point scatterers do not appear to be available in the literature, and we are currently extending the techniques developed in this paper to study problems in which the size of the individual scatterers is not necessarily small, and to include boundary conditions other than those of Dirichlet type.

Appendix A. Integral representations for Hankel functions. We start from the integral representation, valid for $0<\theta<\pi$ (i.e., $y>0$ ),

$$
H_{0}(k r)=-\frac{\mathrm{i}}{\pi} \int_{-\infty}^{\infty+\mathrm{i} \pi} \mathrm{e}^{\mathrm{i} k x \cosh \alpha} \mathrm{e}^{k y \sinh \alpha} \mathrm{d} \alpha .
$$

This integral can be converted into a single integral along the real axis. We first split the integral into three parts, namely $(-\infty, 0),(0, \mathrm{i} \pi)$, and $(\mathrm{i} \pi, \infty+\mathrm{i} \pi)$, and make the substitutions $\alpha=-\operatorname{arccosh} t, \alpha=\mathrm{i} \arccos t$, and $\alpha=\mathrm{i} \pi+\operatorname{arccosh}(-t)$, respectively. This leads, noting that $H_{0}(k r)$ is symmetric in $y$, to

$$
H_{0}(k r)=-\frac{\mathrm{i}}{\pi} \int_{-\infty}^{\infty} \frac{\mathrm{e}^{-k \gamma(t)|y|}}{\gamma(t)} \mathrm{e}^{\mathrm{i} k x t} \mathrm{~d} t
$$

valid for all $y$, where

$$
\gamma(t)= \begin{cases}-\mathrm{i} \sqrt{1-t^{2}}, & |t| \leq 1 \\ \sqrt{t^{2}-1}, & |t|>1\end{cases}
$$

Appendix B. Summation formulas. We can define two generalized functions by

$$
\sum_{m=1}^{\infty} \cos m u=-\frac{1}{2}+\pi \sum_{m=-\infty}^{\infty} \delta(u-2 m \pi), \quad \sum_{m=1}^{\infty} \sin m u=\frac{1}{2} \cot \frac{1}{2} u
$$

(see [9, section 2.4] for more details), from which we can construct the generalized functions

$$
\sum_{m=-\infty}^{\infty} \mathrm{e}^{ \pm \mathrm{i} m u}=2 \pi \sum_{m=-\infty}^{\infty} \delta(u-2 m \pi)
$$

and

$$
\sum_{m=0}^{\infty} \mathrm{e}^{ \pm \mathrm{i} m u}=\frac{1}{1-\mathrm{e}^{ \pm \mathrm{i} u}}+\pi \sum_{m=-\infty}^{\infty} \delta(u-2 m \pi) .
$$


Hence

$$
\sum_{m=-\infty}^{\infty} \int_{-\infty}^{\infty} f(u) \mathrm{e}^{-\mathrm{i} m u} \mathrm{~d} u=2 \pi \sum_{m=-\infty}^{\infty} f(2 m \pi)
$$

which is the Poisson summation formula, and

$$
\sum_{m=0}^{\infty} \int_{-\infty}^{\infty} f(u) \mathrm{e}^{-\mathrm{i} m u} \mathrm{~d} u=f_{-\infty}^{\infty} \frac{f(u)}{1-\mathrm{e}^{-\mathrm{i} u}} \mathrm{~d} u+\pi \sum_{m=-\infty}^{\infty} f(2 m \pi)=f_{-\infty}^{\infty} \frac{f(u)}{1-\mathrm{e}^{-\mathrm{i} u} \mathrm{~d} u}
$$

where the notation means that the contour passes below the poles of the integrand.

Appendix C. Schlömilch series. The quantity $\sigma(\psi)$ is defined by (3.6). An alternative representation is

$$
\sigma(\psi)=-1-\frac{2 \mathrm{i}}{\pi}\left(C+\ln \frac{k s}{4 \pi}\right)+\frac{2}{k s \sin \psi_{0}}+\sum_{\substack{m=-\infty \\ m \neq 0}}^{\infty}\left(\frac{2}{k s \sin \psi_{m}}+\frac{\mathrm{i}}{\pi|m|}\right)
$$

where $C \approx 0.5772$ is Euler's constant. The efficient computation of this series is discussed in [16].

Another important series is

$$
S=\sum_{m=1}^{\infty} \mathrm{e}^{-\mathrm{i} m \beta s} H_{0}(k m s)
$$

To derive an alternative representation more convenient for computation we write

$$
\begin{aligned}
2 S-\sigma & =\sum_{m=1}^{\infty}\left(\mathrm{e}^{-\mathrm{i} m \beta s}-\mathrm{e}^{\mathrm{i} m \beta s}\right) H_{0}(k m s) \\
& =\sum_{m=-\infty}^{\infty}\left(\mathrm{e}^{-\mathrm{i}|m| \beta s}-\mathrm{e}^{-\mathrm{i} m \beta s}\right) H_{0}(k|m| s)=\sum_{m=-\infty}^{\infty} f(2 m \pi),
\end{aligned}
$$

where

$$
f(u)=\left(\mathrm{e}^{-\mathrm{i}|u| \beta s / 2 \pi}-\mathrm{e}^{-\mathrm{i} u \beta s / 2 \pi}\right) H_{0}(k|u| s / 2 \pi) .
$$

The Poisson summation formula (B.1) then gives

$$
\begin{aligned}
2 S-\sigma & =\frac{1}{2 \pi} \sum_{m=-\infty}^{\infty} \int_{-\infty}^{\infty}\left(\mathrm{e}^{-\mathrm{i}|u| \beta s / 2 \pi}-\mathrm{e}^{-\mathrm{i} u \beta s / 2 \pi}\right) H_{0}(k|u| s / 2 \pi) \mathrm{e}^{-\mathrm{i} m u} \mathrm{~d} u \\
& =\frac{1}{s} \sum_{m=-\infty}^{\infty} \int_{-\infty}^{\infty}\left(\mathrm{e}^{-\mathrm{i}|v| \beta}-\mathrm{e}^{-\mathrm{i} v \beta}\right) H_{0}(k|v|) \mathrm{e}^{-\mathrm{i} m 2 \pi v / s} \mathrm{~d} v \\
& =\frac{1}{s} \sum_{m=-\infty}^{\infty} \int_{0}^{\infty}\left(\mathrm{e}^{-\mathrm{i} v \beta_{-m}}-\mathrm{e}^{\mathrm{i} v \beta_{m}}\right) H_{0}(k v) \mathrm{d} v \\
& =\frac{4}{\pi k s}\left(\frac{\frac{1}{2} \pi-\psi}{\sin \psi}+\sum_{m=1}^{\infty}\left[\frac{\frac{1}{2} \pi-\psi_{m}}{\sin \psi_{m}}+\frac{\frac{1}{2} \pi-\psi_{-m}}{\sin \psi_{-m}}\right]\right)
\end{aligned}
$$


where we have used [10, equations $6.671(7),(11)]$. Note that

$$
\frac{\frac{1}{2} \pi-\psi_{m}}{\sin \psi_{m}}+\frac{\frac{1}{2} \pi-\psi_{-m}}{\sin \psi_{-m}} \sim \frac{\beta k s^{2}}{4 m^{2} \pi^{2}}\left(\pi \mathrm{i}-2-2 \ln \left(\frac{4 m \pi}{k s}\right)\right)+O\left(m^{-3}\right) \quad \text { as } m \rightarrow \infty .
$$

As $\psi_{p} \rightarrow 0$ for some integer $p$,

$$
S \sim 2\left(k s \sin \psi_{p}\right)^{-1},
$$

but as $\psi_{p} \rightarrow \pi, S$ is bounded since the singularity in the sum in (C.2) exactly cancels that in $\sigma$.

Appendix D. Asymptotics of a sum. Consider the sum

$$
S_{p}=\sum_{j=p+1}^{\infty} \frac{\mathrm{e}^{\mathrm{i} j \theta}}{j^{1 / 2}} .
$$

Denote by $\Gamma_{p}$ the contour which runs from $\mathrm{i}\left(p+\frac{1}{2}\right)-\infty$ to $\mathrm{i}\left(p+\frac{1}{2}\right)+\infty$ and is closed in the upper half-plane. Then, provided $0<\theta<2 \pi$,

$$
\begin{aligned}
S_{p} & =\frac{1}{2 \pi \mathrm{i}}\left\{-2 \pi \mathrm{i}^{\frac{1}{2}} \int_{\Gamma_{p}} \frac{\mathrm{e}^{\theta t} \mathrm{~d} t}{t^{\frac{1}{2}}\left(1-\mathrm{e}^{2 \pi t}\right)}\right\}=-\frac{1}{\mathrm{i}^{\frac{1}{2}}} \int_{\mathrm{i}\left(p+\frac{1}{2}\right)-\infty}^{\mathrm{i}\left(p+\frac{1}{2}\right)+\infty} \frac{\mathrm{e}^{\theta t} \mathrm{~d} t}{t^{\frac{1}{2}}\left(1-\mathrm{e}^{2 \pi t}\right)} \\
& =-\frac{\mathrm{e}^{\mathrm{i} \theta\left(p+\frac{1}{2}\right)}}{\mathrm{i}^{\frac{1}{2}}} \int_{-\infty}^{\infty} \frac{\mathrm{e}^{\theta u} \mathrm{~d} u}{\left[u+\mathrm{i}\left(p+\frac{1}{2}\right)\right]^{\frac{1}{2}}\left(1+\mathrm{e}^{2 \pi u}\right)} \\
& \sim \frac{\mathrm{i} \mathrm{e}^{\mathrm{i} \theta\left(p+\frac{1}{2}\right)}}{p^{\frac{1}{2}}} \int_{-\infty}^{\infty} \frac{\mathrm{e}^{\theta u} \mathrm{~d} u}{1+\mathrm{e}^{2 \pi u}}=\frac{\mathrm{i} \mathrm{e}^{\mathrm{i} \theta\left(p+\frac{1}{2}\right)}}{2 p^{\frac{1}{2}} \sin \frac{1}{2} \theta}=\frac{-p^{-\frac{1}{2}} \mathrm{e}^{\mathrm{i} \theta p}}{1-\mathrm{e}^{-\frac{\mathrm{i} \theta}{2}}} \quad \text { as } p \rightarrow \infty .
\end{aligned}
$$

If $2 m \pi<\theta<2(m+1) \pi$,

$$
\sum_{j=p+1}^{\infty} \frac{\mathrm{e}^{\mathrm{i} j \theta}}{j^{\frac{1}{2}}}=\sum_{j=p+1}^{\infty} \frac{\mathrm{e}^{\mathrm{i} j(\theta-2 m \pi)}}{j^{\frac{1}{2}}} \sim \frac{-p^{-\frac{1}{2}} \mathrm{e}^{\mathrm{i} \theta p}}{1-(-1)^{m} \mathrm{e}^{-\frac{\mathrm{i} \theta}{2}}} \quad \text { as } p \rightarrow \infty .
$$

Appendix E. Inversion of symmetric Toeplitz matrices. Each of (4.2), (4.3), and (5.8) is of the form

$$
\sum_{m=0}^{\infty} T_{n m} X_{m}=R_{n}, \quad n=0,1,2, \ldots
$$

i.e., $\mathbf{T} X=R$, where $\mathbf{T}$ is a Toeplitz matrix whose elements are given by $T_{n m}=$ $T_{m n}=t_{n-m}$ with

$$
t_{m}= \begin{cases}1, & m=0, \\ -f_{0} H_{0}(k|m| s), & \text { otherwise. }\end{cases}
$$

The matrix $\mathbf{T}$ can be inverted using the discrete Wiener-Hopf technique; the symmetry of $\mathbf{T}$ is not required for this approach but it simplifies the analysis. Here we follow the method as described in [27]. No fully rigorous theory appears to exist which includes the particular matrix $\mathbf{T}$ that occurs in our problem. If the elements of the matrix satisfied $\sum\left|t_{m}\right|<\infty$, or the function $\sum t_{m} \exp (\operatorname{im} \theta)<\infty$ for all $\theta \in(-\pi, \pi)$, 
then we could appeal to the general theory given in [4], [15, section 13]. It is assumed throughout this appendix that we are dealing with a nonresonant case.

First we rewrite (E.1) as

$$
\sum_{m=-\infty}^{\infty} t_{n-m} X_{m}^{+}=R_{n}^{+}+R_{n}^{-}, \quad n=0, \pm 1, \pm 2, \ldots
$$

where $X_{n}^{+}$and $R_{n}^{+}$are equal to $X_{n}$ and $R_{n}$, respectively, when $n \geq 0$, and are zero if $n<0$ and $R_{n}^{-}=0$ for $n \geq 0$, but are otherwise unknown. If we multiply the $n$th equation by $z^{n}$ and then sum over $n$, we get, after writing $n=m+\nu$ on the left-hand side,

$$
\sum_{\nu=-\infty}^{\infty} \sum_{m=-\infty}^{\infty} t_{\nu} X_{m}^{+} z^{m+\nu}=\sum_{n=-\infty}^{\infty} R_{n}^{+} z^{n}+\sum_{n=-\infty}^{\infty} R_{n}^{-} z^{n},
$$

which can be written

$$
K(z) X_{+}(z)=R_{+}(z)+R_{-}(z)
$$

where $X_{+}(z)=\sum_{n=-\infty}^{\infty} X_{n}^{+} z^{n}=\sum_{n=0}^{\infty} X_{n} z^{n}, R_{+}(z)=\sum_{n=0}^{\infty} R_{n} z^{n}, R_{-}(z)=$ $\sum_{n=-\infty}^{-1} R_{n}^{-} z^{n}$, and

$$
K(z)=\sum_{\nu=-\infty}^{\infty} t_{\nu} z^{\nu}=1-f_{0} \sum_{\substack{\nu=-\infty \\ \nu \neq 0}}^{\infty} H_{0}(k s|\nu|) z^{\nu}
$$

Note that

$$
K\left(\mathrm{e}^{ \pm \mathrm{i} k s \cos \theta}\right)=1-f_{0} \sigma(\theta),
$$

where $\sigma(\theta)$ is given by either (3.6) or (C.1), and, in particular, $K\left(\mathrm{e}^{ \pm \mathrm{i} \beta s}\right)=-f_{0} \mathcal{K}$, where $\mathcal{K}$ is defined by (3.7). Here $X_{+}(z)$ is analytic in some disk centered on the origin, and it is reasonable to assume that the radius of convergence is greater than or equal to one. Similar remarks pertain to $R_{+}(z)$. On the other hand, we can assume that $R_{-}(z)$ is analytic in the region exterior to the unit disk. One device that can be used in scattering problems is to let the wavenumber $k$ have a small positive imaginary part, which is equivalent to allowing for a small amount of dissipation in the acoustic medium. This will ensure that the plus functions are analytic for $|z|<\rho_{2}$ and the minus functions are analytic for $|z|>\rho_{1}$, with $\rho_{1}<1<\rho_{2}$. The solution is then obtained by letting the imaginary part of $k$ tend to zero at the end of the calculation. This also takes care of the fact that in our case $K(z)$ actually has singularities on the unit circle, namely inverse square-root branch points at $z=\exp ( \pm \mathrm{i} k s)$.

The solution method is based on a factorization $K(z)=K_{+}(z) K_{-}(z)$, where $K_{+}(z)$ (resp., $K_{-}(z)$ ) is analytic and nonzero inside (resp., outside) and on $|z|=1$. Given such a factorization we have $\ln K(z)=\ln K_{+}(z)+\ln K_{-}(z)=q_{+}(z)+q_{-}(z)$, say, where $q_{+}(z)$ (resp., $\left.q_{-}(z)\right)$ is analytic inside (resp., outside) and on $|z|=1$. From Cauchy's integral formula, writing $q=q_{+}+q_{-}$,

$$
q_{-}(z)=q_{-}(\infty)-\frac{1}{2 \pi \mathrm{i}} \oint_{|\zeta|=1} \frac{q(\zeta)}{\zeta-z} \mathrm{~d} \zeta, \quad|z|>1
$$


Here we must assume that it is possible to choose a single-valued branch of $\ln K(z)$ in some neighborhood of the unit circle. Note that $K(z)=K(1 / z)$, so we can normalize the factorization by requiring that $K_{+}(z)=K_{-}(1 / z)$, in which case $q_{+}(z)=q_{-}(1 / z)$. It follows that

$$
q_{+}(z)=q_{+}(0)-\frac{1}{2 \pi \mathrm{i}} \oint_{|\zeta|=1} \frac{z q(\zeta)}{z \zeta-1} \mathrm{~d} \zeta, \quad|z|<1 .
$$

The required factorization of $K(z)$ (which is unique) is thus given by

$$
\begin{array}{ll}
K_{+}(z)=\frac{1}{\lambda_{0}} \exp \left(\frac{1}{2 \pi \mathrm{i}} \oint_{|\zeta|=1} \frac{z \ln K(\zeta)}{1-z \zeta} \mathrm{d} \zeta\right), & |z|<1, \\
K_{-}(z)=\frac{1}{\lambda_{0}} \exp \left(\frac{1}{2 \pi \mathrm{i}} \oint_{|\zeta|=1} \frac{\ln K(\zeta)}{z-\zeta} \mathrm{d} \zeta\right), & |z|>1,
\end{array}
$$

where $\lambda_{0}=\mathrm{e}^{-q_{+}(0)}$. However, from Cauchy's integral formula

$$
q_{+}(0)=\frac{1}{2 \pi \mathrm{i}} \oint_{|z|=1} \frac{q_{+}(z)}{z} \mathrm{~d} z=\frac{1}{4 \pi \mathrm{i}} \oint_{|z|=1} \frac{q_{+}(z)+q_{-}(z)}{z} \mathrm{~d} z
$$

and thus we have

$$
\lambda_{0}=\exp \left(-\frac{1}{4 \pi \mathrm{i}} \oint_{|z|=1} \frac{\ln K(z)}{z} \mathrm{~d} z\right) .
$$

With this factorization we can rearrange (E.2) as follows:

$$
K_{+} X_{+}-\left(\frac{R_{+}}{K_{-}}\right)_{+}=\left(\frac{R_{+}}{K_{-}}\right)_{-}+\frac{R_{-}}{K_{-}}
$$

in which we have further separated the function $R_{+}(z) / K_{-}(z)$ into the sum of a function analytic inside $|z|=1$ and one analytic outside this circle. Liouville's theorem then implies that both sides must equal a constant. The sum-split of $R_{+} / K_{-}$is performed so that $\left(R_{+} / K_{-}\right)_{-}$tends to zero as $z \rightarrow \infty$. We also have $R_{-} / K_{-} \rightarrow 0$ since $K_{-}(z)$ tends to a nonzero constant in this limit, and so both sides of (E.5) must in fact be zero.

We have thus established that $X_{+}=\left(R_{+} / K_{-}\right)_{+} / K_{+}$and hence that

$$
X_{m}^{+}=\frac{1}{2 \pi \mathrm{i}} \oint_{|z|=1} \frac{\left(R_{+} / K_{-}\right)_{+}(z)}{K_{+}(z)} z^{-m-1} \mathrm{~d} z
$$

Now $\left(R_{+} / K_{-}\right)_{+}(z)=\sum_{n=0}^{\infty} R_{n}\left(z^{n} / K_{-}\right)_{+}(z)$ and

$$
\left(\frac{z^{n}}{K_{-}}\right)_{+}(z)=\sum_{j=0}^{\infty} a_{j}^{(n)} z^{j} \quad \text { with } a_{j}^{(n)}=\frac{1}{2 \pi \mathrm{i}} \oint_{|z|=1} \frac{z^{n-j-1}}{K_{-}(z)} \mathrm{d} z,
$$

the final integral being zero if $j>n$, since then the integrand is regular and nonzero for $|z|>1$ and decays at infinity faster than $1 / z$. Thus we have

$$
X_{m}^{+}=\frac{1}{2 \pi \mathrm{i}} \sum_{n=0}^{\infty} R_{n} \oint_{|z|=1} \sum_{j=0}^{n} a_{j}^{(n)} \frac{z^{j-m-1}}{K_{+}(z)} \mathrm{d} z .
$$


Now if we define $\lambda_{\mu}=a_{n-\mu}^{(n)}$ (with $\lambda_{\mu}=0$ if $\mu<0$ ), then

$$
\lambda_{\mu}=\frac{1}{2 \pi \mathrm{i}} \oint_{|z|=1} \frac{z^{\mu-1}}{K_{-}(z)} \mathrm{d} z,
$$

and so $\lambda_{\mu}, \mu=0,1,2, \ldots$, are the coefficients in the Laurent series for $1 / K_{-}(z)$, i.e., $\left[K_{-}(z)\right]^{-1}=\sum_{\mu=0}^{\infty} \lambda_{\mu} z^{-\mu}$, from which

$$
\frac{1}{K_{+}(z)}=\frac{1}{K_{-}(1 / z)}=\sum_{\mu=0}^{\infty} \lambda_{\mu} z^{\mu} .
$$

Note that $\lambda_{0}=1 / K_{+}(0)$, in agreement with (E.4). The coefficients $\lambda_{\mu}$ can be calculated without knowledge of the functions $K_{ \pm}$since

$$
\lambda_{\mu}=\frac{1}{\mu !} \frac{\mathrm{d}^{\mu}}{\mathrm{d} z^{\mu}}\left[\frac{1}{K_{+}(z)}\right]_{z=0},
$$

and the right-hand side can be evaluated from (E.4) in terms of the weakly singular integrals

$$
\int_{-\pi}^{\pi} \mathrm{e}^{-\mathrm{i} m \theta} \ln \left[K\left(\mathrm{e}^{\mathrm{i} \theta}\right)\right] \mathrm{d} \theta, \quad m=0,1, \ldots
$$

In order to compute $K$ on the unit circle we use (E.3) and (C.1).

The presence of square-root singularities in $K(z)$ at $z=\exp ( \pm \mathrm{i} k s)$ implies, after letting the imaginary part of $k$ tend to zero, a singularity in $K_{+}(z)$ at $z=\exp (-\mathrm{i} k s)$, i.e.,

$$
\left[K_{+}\left(\mathrm{e}^{-\mathrm{i} k s}\right)\right]^{-1}=\sum_{\mu=0}^{\infty} \lambda_{\mu} \mathrm{e}^{-\mathrm{i} \mu k s}=0 .
$$

The function $\left[K_{+}(z)\right]^{-1}$ is smooth everywhere on the unit circle except at the point $z=\exp (-\mathrm{i} k s)$, where its derivative has a square-root singularity. We thus expect (see $[25$, p. 441]) that

$$
\lambda_{\mu}=O\left(\mu^{-3 / 2}\right) \quad \text { as } \mu \rightarrow \infty .
$$

If (E.7) is substituted into (E.6), we get

$$
X_{m}^{+}=\frac{1}{2 \pi \mathrm{i}} \sum_{n=0}^{\infty} R_{n} \oint_{|z|=1} \sum_{j=0}^{n} \lambda_{n-j} z^{j-m-1} \sum_{\mu=0}^{\infty} \lambda_{\mu} z^{\mu} \mathrm{d} z=\sum_{n=0}^{\infty} R_{n} \sum_{j=0}^{\min (n, m)} \lambda_{n-j} \lambda_{m-j} .
$$

We have thus shown that the elements of the (symmetric) inverse matrix $\mathbf{T}^{-1}$ (written $T_{m n}^{-1}, m \geq 0, n \geq 0$ ) are given by

$$
T_{m n}^{-1}=\sum_{j=0}^{\min (m, n)} \lambda_{n-j} \lambda_{m-j} .
$$

The final expression in (E.9) can be rearranged to give

$$
X_{m}=\sum_{p=0}^{\infty} \sum_{q=0}^{m} \lambda_{p} \lambda_{q} R_{m+p-q}
$$


The method of Hills and Karp [12]. For the particular case of (4.2), we have $R_{n}=f_{0} I_{n}$ and so

$$
R_{+}(z)=\sum_{n=0}^{\infty} R_{n} z^{n}=\frac{f_{0}}{1-z \mathrm{e}^{\mathrm{i} \beta s}} .
$$

This is the analytic continuation of the series into the entire complex plane except the point $z=\exp (-\mathrm{i} \beta s)$. Note that, assuming $k$ to have a positive imaginary part, the singularity at $z=\exp (-\mathrm{i} \beta s)$ is exterior to the unit circle. With $A_{+}(z)=\sum_{n=0}^{\infty} A_{n} z^{n}$ we then have

$$
K(z) A_{+}(z)=\frac{f_{0}}{1-z \mathrm{e}^{\mathrm{i} \beta s}}+R_{-}(z) .
$$

Equation (E.11) is $[12,(3.1-3)]$. It is a single equation for two unknown functions, namely $A_{+}(z)$ and $R_{-}(z)$.

The split into plus and minus functions can now be carried out more simply than in the general case. We have

$$
K_{+}(z) A_{+}(z)-\frac{f_{0}}{K_{-}\left(\mathrm{e}^{-\mathrm{i} \beta s}\right)\left(1-z \mathrm{e}^{\mathrm{i} \beta s}\right)}=\frac{f_{0}}{\left(1-z \mathrm{e}^{\mathrm{i} \beta s}\right)}\left(\frac{1}{K_{-}(z)}-\frac{1}{K_{-}\left(\mathrm{e}^{-\mathrm{i} \beta s}\right)}\right)+\frac{R_{-}(z)}{K_{-}(z)},
$$

and Liouville's theorem shows that both sides are zero so that

$$
A_{+}(z)=\frac{f_{0}}{K_{+}(z) K_{-}\left(\mathrm{e}^{-\mathrm{i} \beta s}\right)\left(1-z \mathrm{e}^{\mathrm{i} \beta s}\right)} .
$$

It follows that

$$
A_{m}=\frac{f_{0} I_{m}}{K_{-}\left(\mathrm{e}^{-\mathrm{i} \beta s}\right)} \sum_{q=0}^{m} \lambda_{q} I_{-q}=f_{0} I_{m} \sum_{p=0}^{\infty} \lambda_{p} I_{p} \sum_{q=0}^{m} \lambda_{q} I_{-q}
$$

in agreement with (E.10).

Now, since $A_{n}=I_{n} B_{0}+C_{n}$ and $B_{0}=-1 / \mathcal{K}=f_{0} / K(\exp ( \pm \mathrm{i} \beta s))$,

$$
\sum_{n=0}^{\infty} C_{n} z^{n} \equiv C_{+}(z)=\frac{f_{0}}{K_{-}\left(\mathrm{e}^{-\mathrm{i} \beta s}\right)\left(1-z \mathrm{e}^{\mathrm{i} \beta s}\right)}\left(\frac{1}{K_{+}(z)}-\frac{1}{K_{+}\left(\mathrm{e}^{-\mathrm{i} \beta s}\right)}\right) .
$$

The coefficients $C_{n}$ decay at the same rate as $\lambda_{n}$, i.e.,

$$
C_{n}=O\left(n^{-\frac{3}{2}}\right) \quad \text { as } n \rightarrow \infty,
$$

and for exactly the same reasons. This decay rate for the edge effects was noted in [20, equation 76]. From (E.8), we have

$$
C_{+}\left(\mathrm{e}^{-\mathrm{i} k s}\right)=-\frac{f_{0}}{K\left(\mathrm{e}^{-\mathrm{i} \beta s}\right)\left(1-\mathrm{e}^{\mathrm{i}(\beta-k) s}\right)},
$$

or equivalently,

$$
\sum_{n=0}^{\infty} C_{n} \mathrm{e}^{-\mathrm{i} n k s}=\frac{1}{\mathcal{K}\left(1-\mathrm{e}^{\mathrm{i} k s(\cos \psi-1)}\right)}
$$




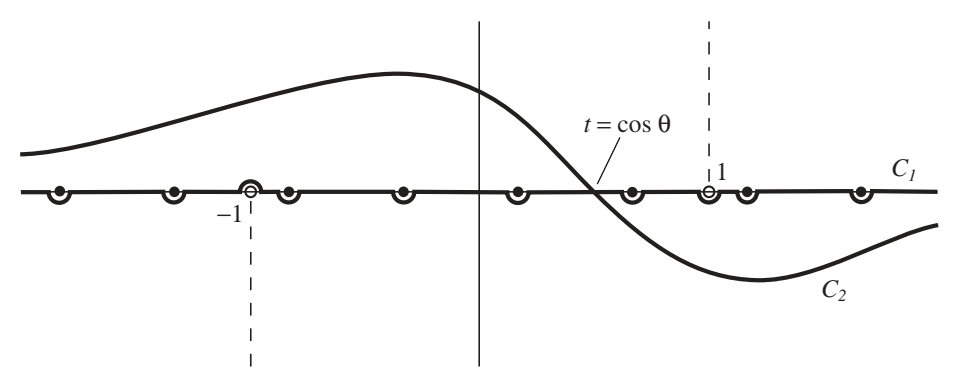

FIG. F.1. Contours $C_{1}$ and $C_{2}$. The dashed lines are branch cuts for the function $\gamma(t)$, and the solid circles are zeros of $1-\mathrm{e}^{\mathrm{is}(\beta-k t)}$ (i.e., $\left.t=\beta_{m} / k\right)$.

Appendix F. Asymptotics of an integral. Consider the integral

$$
I(k r)=f_{-\infty}^{\infty} \frac{\mathrm{e}^{-k \gamma(t) y} \mathrm{e}^{\mathrm{i} k x t} f(t)}{\gamma(t)\left(1-\mathrm{e}^{\mathrm{i} s(\beta-k t)}\right)} \mathrm{d} t=\int_{C_{1}} \frac{f(t) \mathrm{e}^{k r g(t)}}{\gamma(t)\left(1-\mathrm{e}^{\mathrm{i} s(\beta-k t)}\right)} \mathrm{d} t,
$$

where $y=r \sin \theta>0$,

$$
g(t)=-\gamma(t) \sin \theta+\mathrm{i} t \cos \theta,
$$

and $C_{1}$ is the contour shown in Figure F.1. Here the branch of $\gamma(t)$ (defined for real $t$ by (A.2)) is indicated by the branch cuts shown in the figure, and $f$ is assumed regular throughout the complex $t$-plane. We will assume that $\left|\beta_{m} / k\right| \neq 1$ holds for all $m$ so that none of the poles of the integrand coincide with the branch points of $\gamma$. The function $g$ has one simple saddle point in the complex $t$-plane at $t=\cos \theta$ and

$$
g(\cos \theta)=\mathrm{i}, \quad g^{\prime \prime}(\cos \theta)=-\mathrm{i} / \sin ^{2} \theta .
$$

In order to derive the asymptotics of $I$ for large $k r$ we need to deform the contour $C_{1}$ into the path of steepest descent. This is the curve on which Imag $g=1$, which passes through the saddle point, making an angle $-\pi / 4$ with the positive real $t$-axis. This curve crosses the real axis again at $t=1 / \cos \theta$. In deforming the contour, we pick up contributions from the poles on the real axis over which we pass. Only those poles between -1 and 1 give any contribution in the limit as $k r \rightarrow \infty$, the others leading to exponentially small terms. Hence we can deform the contour back down to the real axis to produce $C_{2}$ without affecting the asymptotics.

Hence, as $k r \rightarrow \infty$,

$$
\begin{aligned}
I(k r) \sim & \int_{C_{2}} \frac{f(t) \mathrm{e}^{k r g(t)}}{\gamma(t)\left(1-\mathrm{e}^{\mathrm{i} s(\beta-k t)}\right)} \mathrm{d} t+2 \pi \mathrm{i} \sum_{\substack{m \in \mathcal{M} \\
\beta_{m}<k \cos \theta}} \frac{f\left(\beta_{m} / k\right) \mathrm{e}^{\mathrm{i} k r \cos \left(\theta-\psi_{m}\right)}}{k s \sin \psi_{m}} \\
\sim & \frac{f(\cos \theta) \mathrm{e}^{k r g(\cos \theta)-\frac{1}{4} \pi \mathrm{i}}}{-\mathrm{i} \sin \theta\left(1-\mathrm{e}^{\mathrm{i} s(\beta-k \cos \theta)}\right)} \sqrt{\frac{2 \pi}{k r\left|g^{\prime \prime}(\cos \theta)\right|}} \\
& +2 \pi \mathrm{i} \sum_{\substack{m \in \mathcal{M} \\
\cos \psi_{m}<\cos \theta}} \frac{f\left(\beta_{m} / k\right) \mathrm{e}^{\mathrm{i} k r \cos \left(\theta-\psi_{m}\right)}}{k s \sin \psi_{m}} \\
= & \frac{\mathrm{i} f(\cos \theta) \mathrm{e}^{\mathrm{i}\left(k r-\frac{1}{4} \pi\right)}}{1-\mathrm{e}^{\mathrm{i} k s(\cos \psi-\cos \theta)}} \sqrt{\frac{2 \pi}{k r}}+2 \pi \mathrm{i} \sum_{\substack{m \in \mathcal{M} \\
\psi_{m}>\theta}} \frac{f\left(\beta_{m} / k\right) \mathrm{e}^{\mathrm{i} k r \cos \left(\theta-\psi_{m}\right)}}{k s \sin \psi_{m}}
\end{aligned}
$$


where we have used $\gamma(\cos \theta)=-i \sin \theta$ and the asymptotics of the integral along the steepest descent contour are given, for example, by [7, equation 4.2.1b]. To obtain the asymptotics valid for $y<0$ we simply replace $\theta$ by $-\theta$ in (F.1). It is implicit in the above that the saddle point of $g$ does not coincide with any of the zeros of $1-\mathrm{e}^{\mathrm{i} s(\beta-k t)}$. In other words, we have assumed that $|\theta| \neq \psi_{m}$ for any integer $m$.

Uniform asymptotics valid as $\psi_{p} \rightarrow|\theta|$ can be obtained; see [7, section 4.4], for example. A lengthy calculation shows that we must add a term

$$
\tilde{I}=\frac{\sqrt{\pi} \mathrm{e}^{\mathrm{i}\left(k r-\frac{1}{4} \pi\right)}}{k s \zeta_{p} \sin \psi_{p}} \operatorname{sgn}\left(|\theta|-\psi_{p}\right) f\left(\beta_{p} / k\right)\left(1+2 \mathrm{i} \zeta_{p} \mathrm{e}^{-\mathrm{i} \zeta_{p}^{2}} F\left(\zeta_{p}\right)\right)
$$

to the right-hand side of (F.1). Here $\zeta_{p}=\sqrt{2 k r} \sin \left(\frac{1}{2}|| \theta\left|-\psi_{p}\right|\right)$, and

$$
F(v)=\int_{v}^{\infty} \mathrm{e}^{\mathrm{i} u^{2}} \mathrm{~d} u \quad\left(0<\arg u<\frac{1}{2} \pi \text { as } u \rightarrow \infty\right)
$$

is a Fresnel integral. Since (see [3, p. 67])

$$
F(v) \sim \frac{\mathrm{i}}{2 v} \mathrm{e}^{\mathrm{i} v^{2}}\left(1+\sum_{n=1}^{\infty} \frac{(2 n-1) ! !}{\left(2 \mathrm{i} v^{2}\right)^{n}}\right) \quad \text { as } v \rightarrow \infty, \quad-\frac{1}{2} \pi<\arg v<\pi,
$$

we have

$$
\tilde{I} \sim-\frac{\sqrt{\pi} \mathrm{e}^{\mathrm{i}\left(k r-\frac{1}{4} \pi\right)}}{2 \mathrm{i} k s \zeta_{p}^{3} \sin \psi_{p}} \operatorname{sgn}\left(|\theta|-\psi_{p}\right) f\left(\beta_{p} / k\right) \quad \text { as } \zeta_{p} \rightarrow \infty .
$$

Appendix G. The quantities $\mathcal{G}_{n}$. From (5.7), we have

$$
\mathcal{G}_{n}=\frac{1}{2 \pi a} \int_{-a}^{a} \int_{-a}^{a} \frac{H_{0}(k|x-\xi-n s|)}{\sqrt{a^{2}-\xi^{2}}} \mathrm{~d} \xi \mathrm{d} x .
$$

For $n=0$, with $k a \ll 1$, we can approximate this by

$$
\begin{aligned}
\mathcal{G}_{0} & \approx \frac{1}{2 \pi^{2} a} \int_{-a}^{a} \int_{-a}^{a} \frac{\pi+2 \mathrm{i}\left(C+\ln \frac{1}{2} k|x-\xi|\right)}{\sqrt{a^{2}-\xi^{2}}} \mathrm{~d} \xi \mathrm{d} x \\
& =1+\frac{2 \mathrm{i} C}{\pi}+\frac{\mathrm{i}}{\pi^{2} a} \int_{-a}^{a} \int_{-a}^{a} \frac{\ln \frac{1}{2} k|x-\xi|}{\sqrt{a^{2}-\xi^{2}}} \mathrm{~d} \xi \mathrm{d} x=1+\frac{2 \mathrm{i}}{\pi}\left(C+\ln \frac{1}{4} k a\right) .
\end{aligned}
$$

In [22] the $O\left((k a)^{2}\right)$ terms in $\mathcal{G}_{0}$ are also evaluated, but this seems to be inconsistent with the level of approximation being used. For $n \neq 0$ we use Neumann's addition theorem [1, equation 9.1.75], which shows that

$$
H_{0}(k|n| s \pm k(x-\xi))=\sum_{m=-\infty}^{\infty} H_{ \pm m}(k|n| s) J_{m}(k(x-\xi)) \approx H_{0}(k|n| s)
$$

since $k a \ll 1$. Hence

$$
\mathcal{G}_{n} \approx H_{0}(k|n| s) .
$$




\section{REFERENCES}

[1] M. Abramowitz and I. A. Stegun, Handbook of Mathematical Functions, Dover Publications, New York, 1965.

[2] C. Aristégui And Y. C. Angel, New results for isotropic point scatterers: Foldy revisited, Wave Motion, 36 (2002), pp. 383-399.

[3] J. J. Bowman, T. B. A. Senior, and P. L. E. Uslenghi, eds., Electromagnetic and Acoustic Scattering by Simple Shapes, revised ed., Hemisphere, New York, 1987.

[4] A. Calderón, F. Spitzer, and H. Widom, Inversion of Toeplitz matrices, Illinois J. Math., 3 (1959), pp. 490-498.

[5] F. Capolino, M. Albani, S. Maci, and L. B. Felsen, Frequency-domain Green's function for a planar periodic semi-infinite phased array-Part I: Truncated Floquet wave formulation, IEEE Trans. Antennas and Propagation, 48 (2000), pp. 67-74.

[6] F. Capolino, M. Albani, S. Maci, and L. B. Felsen, Frequency-domain Green's function for a planar periodic semi-infinite phased array - Part II: Diffracted wave phenomenology, IEEE Trans. Antennas and Propagation, 48 (2000), pp. 75-85.

[7] L. B. Felsen and N. Marcuvitz, Radiation and Scattering of Waves, Prentice-Hall, Englewood Cliffs, NJ, 1973.

[8] L. L. Foldy, The multiple scattering of waves I. General theory of isotropic scattering by randomly distributed scatterers, Phys. Rev., 67 (1945), pp. 107-119.

[9] I. M. Gel'fand And G. E. Shilov, Generalized Functions. Volume 1. Properties and Operations, Academic Press, New York, 1964.

[10] I. S. Gradshteyn And I. M. Ryzhik, Tables of Integrals, Series and Products, 4th ed., Academic Press, New York, 1980.

[11] N. L. Hills, Semi-infinite diffraction gratings. II. Inward resonance, Comm. Pure Appl. Math., 18 (1965), pp. 389-395.

[12] N. L. Hills AND S. N. KarP, Semi-infinite diffraction gratings-I, Comm. Pure Appl. Math., 18 (1965), pp. 203-233.

[13] A. Ishimaru, R. J. Coe, G. E. Miller, and W. P. Geren, Finite periodic structure approach to large scanning array problems, IEEE Trans. Antennas and Propagation, 33 (1985), pp. $1213-1220$.

[14] R. Kleinman and B. Vainberg, Full low-frequency asymptotic expansion for second-order elliptic equations in two dimensions, Math. Methods Appl. Sci., 17 (1994), pp. 989-1004.

[15] M. G. KREIN, Integral equations on a half-line with kernel depending upon the difference of the arguments, Amer. Math. Soc. Transl. Ser. 2, 22 (1962), pp. 163-288.

[16] C. M. Linton, The Green's function for the two-dimensional Helmholtz equation in periodic domains, J. Engrg. Math., 33 (1998), pp. 377-402.

[17] S. Maci, F. Capolino, and L. B. Felsen, Three-dimensional Green's function for planar rectangular phased dipole arrays, Wave Motion, 34 (2001), pp. 263-279.

[18] R. F. Millar, Plane wave spectra in grating theory. III. Scattering by a semiinfinite grating of identical cylinders, Canad. J. Phys., 42 (1964), pp. 1149-1184.

[19] R. F. Millar, On a non-linear integral equation occurring in diffraction theory, Proc. Cambridge Philos. Soc., 62 (1966), pp. 249-261.

[20] R. F. Millar, Plane wave spectra in grating theory. V. Scattering by a semi-infinite grating of isotropic scatterers, Canad. J. Phys., 44 (1966), pp. 2839-2874.

[21] A. Neto, S. Maci, G. Vecchi, and M. Sabbadini, A truncated Floquet wave diffraction method for the full wave analysis of large phased arrays-Part I: Basic principles and 2-D cases, IEEE Trans. Antennas and Propagation, 48 (2000), pp. 594-600.

[22] M. Nishimoto And H. IkUno, Analysis of electromagnetic wave diffraction by a semi-infinite strip grating and evaluation of end-effects, Progress in Electromagnetics Research, 23 (1999), pp. 39-58.

[23] M. Nishimoto AND H. IKuno, Space-wavenumber analysis of field scattered from a semi-infinite strip grating, Electrical Engineering in Japan, 132 (2000), pp. 1-8.

[24] A. J. Roscoe and R. A. Perrott, Large finite array analysis using infinite array data, IEEE Trans. Antennas and Propagation, 42 (1994), pp. 983-992.

[25] E. C. Titchmarsh, The Theory of Functions, 2nd ed., Oxford University Press, London, 1939.

[26] B. Tomasic And A. Hessel, Analysis of finite arrays - A new approach, IEEE Trans. Antennas and Propagation, 47 (1999), pp. 555-565.

[27] W. WasylkiWskyj, Mutual coupling effects in semi-infinite arrays, IEEE Trans. Antennas and Propagation, 21 (1973), pp. 277-285. 